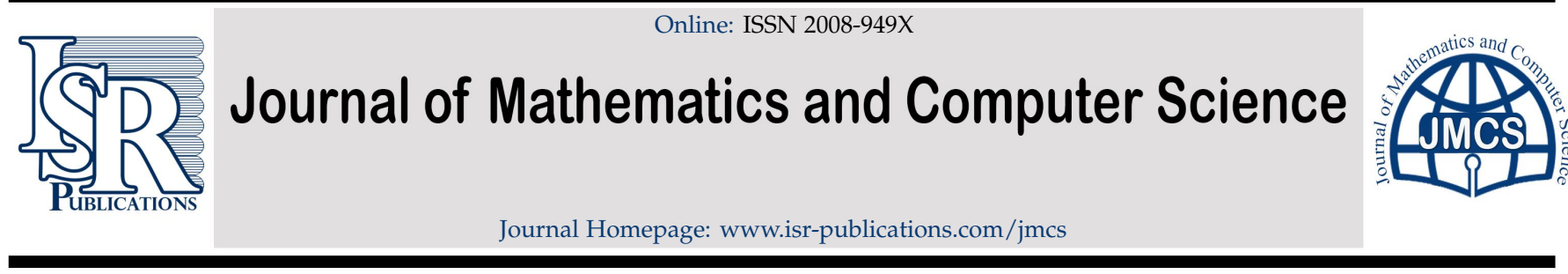

\title{
Oscillatory behavior of second order delay dynamic equa- tions with a sub-linear neutral term on time scales
}

\author{
A. A. Soliman, A. M. Hassan*, S. E. Affan \\ Department of Mathematics, Faculty of Science, Benha University, Benha-Kalubia 13518, Egypt.
}

\begin{abstract}
This work concerned with the oscillation of solutions of a class of second order delay dynamic equations with a sub-linear neutral term. The obtained results essentially improve, complement and simplify a number of related ones in the literature. Some examples are given to illustrate our main results.
\end{abstract}

Keywords: Second order, sub-linear neutral term, oscillation, Riccati transformation.

2020 MSC: 34C11, 34K11.

(C)2022 All rights reserved.

\section{Introduction}

The aim of this paper is to study the oscillation problem of a class of second-order non-linear dynamic equations with a sub-linear neutral term

$$
\left(r(t) z^{\Delta}(t)\right)^{\Delta}+q(t) x^{\beta}(\delta(t))=0, \quad t \geqslant t_{0},
$$

where $0<\alpha \leqslant 1, \beta$ are ratios of odd positive integers and $z(t):=x(t)+p(t) x^{\alpha}(\tau(t))$. Throughout, the following assumptions are satisfied.

(H1) $r \in C_{r d}^{1}\left(\left[t_{0}, \infty\right)_{\mathbb{T}},(0, \infty)\right), R(t)=\int_{t_{0}}^{t} \frac{1}{r(s)} \Delta s$, and $\hat{R}(t)=\int_{t}^{\infty} \frac{1}{r(s)} \Delta s$

(H2) $p, q \in \mathrm{C}_{\mathrm{rd}}\left(\left[\mathrm{t}_{0}, \infty\right)_{\mathbb{T}},[0, \infty)\right)$ and $\mathrm{q}(\mathrm{t})$ is not eventually zero for sufficiently large $\mathrm{t}$.

(H3) $\tau, \delta \in \mathrm{C}_{\mathrm{rd}}\left(\left[\mathrm{t}_{0}, \infty\right)_{\mathbb{T}}, \mathbb{T}\right), \tau(\mathrm{t}) \leqslant \mathrm{t}, \delta(\mathrm{t}) \leqslant \mathrm{t}$ and $\lim _{\mathrm{t} \rightarrow \infty} \tau(\mathrm{t})=\lim _{\mathrm{t} \rightarrow \infty} \delta(\mathrm{t})=\infty$

By a solution of (1.1), we mean a function $x \in \mathrm{C}_{\mathrm{rd}}\left[\mathrm{T}_{x}, \infty\right)_{\mathbb{T}}, \mathrm{T}_{x} \in\left[\mathrm{t}_{0}, \infty\right)_{\mathbb{T}}$ which has the property $r(t) z^{\Delta}(t) \in C_{r d}^{1}\left[T_{x}, \infty\right)_{\mathbb{T}}$ and satisfies (1.1) on $\left[T_{x}, \infty\right)_{\mathbb{T}}$. We consider only those solutions $x(t)$ of (1.1) which satisfy $\sup |x(t)|: t \in\left[T_{x}, \infty\right)_{\mathbb{T}}>0$ for all $T \in\left[T_{x}, \infty\right)_{\mathbb{T}}$. We assume that (1.1) possesses such solutions. A solution of (1.1) is called oscillatory if it is neither eventually positive nor eventually negative;

\footnotetext{
*Corresponding author

Email addresses: a_a_soliman@hotmail.com (A. A. Soliman), ahmed.mohamed@fsc.bu.edu.eg (A. M. Hassan),

samy. affan@fsc.bu.edu.eg (S. E. Affan)
}

doi: $10.22436 /$ jmcs.024.02.01

Received: 2020-10-29 Revised: 2020-11-22 Accepted: 2020-12-02 
otherwise, it is termed nonoscillatory. Dynamic equations on time scales have an enormous potential for applications in biology, engineering, economics, physics, neural networks, social sciences, and so forth.

In recent years, there has been a great interest in studying and establishing criteria for the oscillatory and asymptotic behavior of various classes of dynamic equations on time scales, see; [3, 5, 7, 18, 23, 24, 27].

However, it seems that there are no known results for the oscillation of second-order dynamic equations with positive sublinear neutral terms. Also, in special cases $\mathbb{T}=\mathbb{R}$ and $\mathbb{T}=\mathbb{Z}$ there are a few literature present sufficient criteria for the oscillatory behavior of second-order differential equations and difference equations with positive sublinear neutral terms, see; [1, 4, 6, 12-16, 25].

For instance, in the particular case of (1.1) when $\mathbb{T}=\mathbb{R}$, Tamilvanan et al. [25] established sufficient conditions for the oscillation of all solutions of a nonlinear differential equation

$$
\left(a(t)\left(x(t)+p(t) x^{\alpha}(\tau(t))\right)^{\prime}\right)^{\prime}+q(t) x^{\beta}(\sigma(t))=0, t \geqslant t_{0}
$$

where $0<\alpha \leqslant 1$, and $\beta$ are ratios of odd positive integers, under the assumption that $\int_{t_{0}}^{\infty} \frac{d t}{a(t)}=\infty$. The authors in [13] studied a more general equation than (1.2) in the noncanonical case

$$
\left(a\left(y^{\prime}\right)^{\gamma}\right)^{\prime}(t)+q(t) x^{\beta}(\sigma(t))=0, \quad t \geqslant t_{0}>0,
$$

where $y(t):=x(t)+p(t) x^{\alpha}(\tau(t))$, under the condition $A\left(t_{0}\right):=\int_{t_{0}}^{\infty} \frac{1}{a^{1 / \gamma}(s)} d s<\infty$. The authors in [16] made a contribution to oscillation theory of neutral type delay differential equations

$$
\left(a(t)\left(x(t)+p(t) x^{\alpha}(\sigma(t))^{\prime}\right)^{\gamma}\right)^{\prime}+q(t) x^{\beta}(\tau(t))=0, \quad t \geqslant t_{0}
$$

under the condition $A\left(t_{0}\right):=\int_{t_{0}}^{\infty} \frac{1}{a^{1 / \gamma(s)}} d s<\infty$. The authors in [1] established sufficient conditions for the oscillation of a class of second-order differential equations of the form

$$
\left(r(t)\left(x(t)+p(t) x^{\alpha}(\tau(t))\right)^{\prime}\right)^{\prime}+q(t) x(\sigma(t))=0, \quad t \geqslant t_{0}
$$

where $z(t):=x(t)+p(t) x^{\alpha}(\tau(t))$, under the assumptions that $\int_{t_{0}}^{\infty} \frac{d t}{r(t)}=\infty$ and $\int_{t_{0}}^{\infty} \frac{d t}{r(t)}<\infty$.

When $\mathbb{T}=\mathbb{Z}$, in [12] Dharuman et al. concerned with the oscillatory behavior of the nonlinear difference equation with a sub-linear neutral term

$$
\Delta\left(a_{n} \Delta\left(x_{n}+p_{n} x_{n-k}^{\alpha}\right)\right)+q_{n} x_{n+1-l}^{\beta}=0, n \geqslant n_{0},
$$

in the two cases $\sum_{n=n_{0}}^{\infty} \frac{1}{a_{n}}=\infty$ and $\sum_{n=n_{0}}^{\infty} \frac{1}{a_{n}}<\infty$.

In fact, equation (1.1) has numerous applications in mathematical, theoretical, and chemical physics; see, e.g., it arises in a variety of real world problems such as in the study of p-Laplace equations nonNewtonian fluid theory, the turbulentflow of a polytrophic gas in a porous medium, and so forth. So, there has been much research activity concerning oscillatory behavior of various classes of differential equations. We refer the reader to $[2,8,19-21]$.

Motivated by this observation, our aim in this paper is to present sufficient conditions for the oscillatory behavior of solutions of (1.1), under the conditions either

$$
\int_{\mathrm{t}_{0}}^{\infty} \frac{1}{\mathrm{r}(\mathrm{s})} \Delta \mathrm{s}=\infty, \quad \text { or } \quad \int_{\mathrm{t}_{0}}^{\infty} \frac{1}{\mathrm{r}(\mathrm{s})} \Delta \mathrm{s}<\infty .
$$

The obtained results unify, improve and extend some results in the literature. In what follows, all functional inequalities are assumed to hold eventually. Without loss of generality, we can deal only with eventually positive solutions of (1.1). 


\section{Main results}

For simplicity, through this paper for some $M>0$ and $C>0$ we consider the following

$$
\mathrm{Q}(\mathrm{t})=1-\mathrm{p}(\mathrm{t})\left(\frac{\mathrm{R}(\sigma(\mathrm{t}))}{\mathrm{MR}(\mathrm{t})}\right)^{1-\alpha}, \quad \mathrm{P}(\mathrm{t}):=1-\mathrm{C}^{\alpha-1} \mathrm{p}(\mathrm{t}) \frac{\hat{\mathrm{R}}^{\alpha}(\tau(\mathrm{t}))}{\hat{R}(\sigma(t))}, \quad \varphi(\mathrm{t}):=\mathrm{q}(\mathrm{t}) \mathrm{P}^{\beta}(\delta(\mathrm{t})) .
$$

Lemma 2.1 ([26]). Assume that $\beta \geqslant 1$ and $a, b \in[0, \infty)$. Then

$$
a^{\beta}+b^{\beta} \geqslant \frac{1}{2^{\beta-1}}(a+b)^{\beta} .
$$

Lemma 2.2 ([26]). Assume that $0<\beta \leqslant 1$ and $\mathrm{a}, \mathrm{b} \in[0, \infty)$. Then

$$
a^{\beta}+b^{\beta} \geqslant(a+b)^{\beta} \text {. }
$$

Theorem 2.3 ([10]). Assume that $v: \mathbb{T} \rightarrow \mathbb{R}$ is strictly increasing and $\tilde{\mathbb{T}}:=v(\mathbb{T})$ is a time scale. Let $y: \tilde{\mathbb{T}} \rightarrow \mathbb{R}$. If $\mathrm{y}^{\tilde{\Delta}}(v(\mathrm{t}))$ and $v^{\Delta}(\mathrm{t})$ exist for $\mathrm{t} \in \mathbb{T}^{\mathrm{k}}$, then

$$
(y \circ v)^{\Delta}(t)=y^{\tilde{\Delta}}(v(t)) v^{\Delta}(t)
$$

Where $\tilde{\Delta}$ denotes to the derivative on $\tilde{\mathbb{T}}$.

First, we consider

$$
\int_{\mathrm{t}_{0}}^{\infty} \frac{1}{\mathrm{r}(\mathrm{s})} \Delta \mathrm{s}=\infty
$$

In what follows, we present known results, which will be useful in the proof of our main results.

Lemma 2.4. Let (H1)-(H3) and (2.1) hold. If $x(t)$ is an eventually positive solution of (1.1), then $z(t)$ satisfies

(I) $z(\mathrm{t})>0, z^{\Delta}(\mathrm{t})>0$, and $\left(\mathrm{r}(\mathrm{t}) z^{\Delta}(\mathrm{t})\right)^{\Delta}<0, \mathrm{t} \geqslant \mathrm{t}_{1} \geqslant \mathrm{t}_{0}$;

(II) $\frac{z(t)}{R(t)}$ is decreasing for $t \geqslant t_{1}$.

Proof. Since $x$ is an eventually positive solution of (1.1), then by (H2) and (H3) there exists $t_{1} \in\left[t_{0}, \infty\right)_{\mathbb{T}}$ such that $x(t)>0, x(\delta(t))>0$ and $x(\tau(t))>0$ for all $t \in\left[t_{0}, \infty\right)_{\mathbb{T}}$. Now, from (1.1) we have

$$
\left(\mathrm{r}(\mathrm{t}) z^{\Delta}(\mathrm{t})\right)^{\Delta} \leqslant-\mathrm{q}(\mathrm{t}) \chi^{\beta}(\delta(\mathrm{t})) .
$$

Hence $\left(r(t) z^{\Delta}(t)\right)$ is a nonincreasing function and is eventually of one sign. We claim that $z^{\Delta}(t)>0$ for all $t \in\left[t_{1}, \infty\right)_{\mathbb{T}}$. If not, then there exists $t_{2} \in\left[t_{1}, \infty\right)_{\mathbb{T}}$ such that $z^{\Delta}(t) \leqslant 0$ for all $t_{2} \in\left[t_{1}, \infty\right)_{\mathbb{T}}$. Since $q$ is not identical to zero for large $t$, we may assume that $z^{\Delta}(t)<0$ for all $t \in\left[t_{2}, \infty\right)_{\mathbb{T}}$. From (2.2), we have

$$
\left(\mathrm{r}(\mathrm{t}) z^{\Delta}(\mathrm{t})\right) \leqslant-\mathrm{c}<0, \quad \text { for all } \mathrm{t} \in\left[\mathrm{t}_{2}, \infty\right)_{\mathbb{T}},
$$

where $c:=\left(r\left(t_{2}\right) z^{\Delta}\left(t_{2}\right)\right)>0$, then

$$
z^{\Delta}(\mathrm{t}) \leqslant \frac{-\mathrm{c}}{\mathrm{r}(\mathrm{t})}
$$

Integrating (2.3) on $\left[t_{2}, t\right) \subset\left[t_{2}, \infty\right)_{\mathbb{T}}$, we obtain

$$
z(t) \leqslant z\left(t_{2}\right)-c \int_{t_{2}}^{t} \frac{\Delta s}{r(s)}, \quad \text { for all } t \in\left[t_{2}, \infty\right)_{\mathbb{T}} .
$$

Letting $t \rightarrow \infty$, then it follows from (2.1) that $\lim _{t \rightarrow \infty} z(t)=-\infty$, which is a contradiction. Then

$$
z(t)>0, z^{\Delta}(t)>0, \text { and }\left(r(t) z^{\Delta}(t)\right)^{\Delta}<0, \quad t \geqslant t_{1} \geqslant t_{0} .
$$


To prove (II), since (I) holds, then for sufficiently large $t_{1}$

$$
z(t) \geqslant \int_{t_{1}}^{t} \frac{r(s) z^{\Delta}(s)}{r(s)} \Delta s \geqslant r(t) R(t) z^{\Delta}(t)
$$

Moreover, by using the last inequality, we get

$$
\left(\frac{z(t)}{R(t)}\right)^{\Delta}=\frac{z^{\Delta}(t) R(t)-z(t) R^{\Delta}(t)}{R(t) R(\sigma(t))}=\frac{r(t) z^{\Delta}(t) R(t)-z(t)}{r(t) R(t) R(\sigma(t))} \leqslant 0 .
$$

This means that $\frac{z(t)}{R(t)}$ is decreasing for $t \geqslant t_{1}$ and completes the proof .

The following theorem present oscillation criteria for (1.1) where $\beta \geqslant 1$.

Theorem 2.5. Assume that (H1)-(H3) and (2.1) hold, and $\delta^{\Delta}>0$. If $\beta \geqslant 1$ and there exists a function $\psi(t) \in$ $\mathrm{C}_{\mathrm{rd}}^{1}\left(\left[\mathrm{t}_{0}, \infty\right)_{\mathbb{T}},(0, \infty)\right)$, such that for sufficiently large $\mathrm{t}_{2} \geqslant \mathrm{t}_{1}$, and for any $\mathrm{M}>0$,

$$
\limsup _{t \rightarrow \infty} \int_{t_{2}}^{t}\left(\psi(s) q(s) Q^{\beta}(\delta(s))-\frac{1}{4} \frac{\left(\psi^{\Delta}(s)\right)^{2} r(\delta(s))}{\beta M^{(\beta-1)} \psi(s) \delta^{\Delta}(s)}\right) \Delta s=\infty,
$$

holds, then (1.1) is oscillatory.

Proof. Let $x(t)$ be a nonoscillatory solution of $(1.1)$ on $\left[t_{0}, \infty\right)_{\mathbb{T}}$ such that $x(t)>0, x(\tau(t))>0$, and $x(\delta(t))>$ 0 for $t \in\left[t_{1}, \infty\right)_{\mathbb{T}}$.

From (1.1), condition (2.1), and since $\left(r(t) z^{\Delta}(t)\right)$ is non-increasing, we can obtain that

$$
r(t) z^{\Delta}(t) \leqslant r(\delta(t)) z^{\Delta}(\delta(t)), \quad t \geqslant t_{1} .
$$

Also since $z^{\Delta}(t)>0$, so there exists a constant $M>0$ such that $z(t) \geqslant M$ for all large $t \geqslant t_{1}$. From definition of $z(t), M$ and since $z(t)$ is increasing, we get

$$
x(t)=z(t)-p(t) x^{\alpha}(\tau(t)) \geqslant z(t)-p(t) z^{\alpha}(\tau(t)) \geqslant z(t)-p(t) z^{\alpha}(t) \geqslant\left(1-p(t) \frac{1}{z^{\alpha-1}(t)}\right) z(t) .
$$

Since $\frac{z(t)}{R(t)}$ is decreasing, we get

$$
x(t) \geqslant\left(1-p(t)\left(\frac{R(\sigma(t))}{M R(t)}\right)^{1-\alpha}\right) z(t) \geqslant Q(t) z(t) .
$$

This with (1.1), leads to

$$
\left[\mathrm{r}(\mathrm{t}) z^{\Delta}(\mathrm{t})\right]^{\Delta} \leqslant-\mathrm{q}(\mathrm{t}) \mathrm{Q}^{\beta}(\delta(\mathrm{t})) z^{\beta}(\delta(\mathrm{t})) .
$$

Define the Riccati substitution

$$
\omega(t)=\psi(t) \frac{r(t) z^{\Delta}(t)}{z^{\beta}(\delta(t))} .
$$

It is clear that $\omega(t)>0$ for $t \geqslant t_{1}$ and

$$
\begin{aligned}
\omega^{\Delta}(t) & =\psi^{\Delta}(t) \frac{r(\sigma(t)) z^{\Delta}(\sigma(t))}{z^{\beta}(\delta(\sigma(t)))}+\psi(t)\left(\frac{r(t) z^{\Delta}(t)}{z^{\beta}(\delta(t))}\right)^{\Delta} \\
& =\psi^{\Delta}(t) \frac{r(\sigma(t)) z^{\Delta}(\sigma(t))}{z^{\beta}(\delta(\sigma(t)))}+\psi(t)\left[\frac{\left[r(t) z^{\Delta}(t)\right]^{\Delta} z^{\beta}(\delta(t))-r(t) z^{\Delta}(t)\left(z^{\beta}(\delta(t))\right)^{\Delta}}{z^{\beta}(\delta(t)) z^{\beta}(\delta(\sigma(t)))}\right] .
\end{aligned}
$$


Applying the Pötzsche chain rule and Theorem 2.3, we get

$$
\begin{aligned}
\left(z^{\beta}(\delta(t))\right)^{\Delta} & =\beta\left\{\int_{0}^{1}\left[z(\delta(t))+h \mu[z(\delta(t))]^{\Delta}\right]^{\beta-1} \mathrm{dh}\right\} z^{\Delta}(\delta(t)) \delta^{\Delta}(\mathrm{t}) \\
& =\beta\left\{\int_{0}^{1}\left[(1-h) z(\delta(t))+h \mu z\left(\delta^{\sigma}(\mathrm{t})\right)\right]^{\beta-1} \mathrm{dh}\right\} z^{\Delta}(\delta(t)) \delta^{\Delta}(\mathrm{t}) \\
& \geqslant \beta z^{\beta-1}\left(\delta^{\sigma}(\mathrm{t})\right) z^{\Delta}(\delta(\mathrm{t})) \delta^{\Delta}(\mathrm{t}) .
\end{aligned}
$$

This with (2.6) and and taking into account that $z^{\Delta}(\mathrm{t})>0$, and $\delta^{\Delta}(\mathrm{t})>0$, leads to

$$
\omega^{\Delta}(t) \leqslant \psi^{\Delta}(t) \frac{r(\sigma(t)) z^{\Delta}(\sigma(t))}{z^{\beta}(\delta(\sigma(t)))}+\psi(t) \frac{\left[r(t) z^{\Delta}(t)\right]^{\Delta}}{z^{\beta}(\delta(\sigma(t)))}-\beta \psi(t) \delta^{\Delta}(t) \frac{r(t) z^{\Delta}(t) z^{\Delta}(\delta(t))}{z^{\beta+1}(\delta(\sigma(t)))} .
$$

Also, since $\left(r(t) z^{\Delta}(t)\right)$ is non-increasing and from definition of $\omega(t)$, we get

$$
\begin{aligned}
\omega^{\Delta}(\mathrm{t}) & \leqslant \psi^{\Delta}(\mathrm{t}) \frac{\mathrm{r}(\sigma(\mathrm{t})) z^{\Delta}(\sigma(\mathrm{t}))}{z^{\beta}(\delta(\sigma(t)))}+\psi(\mathrm{t}) \frac{\left[\mathrm{r}(\mathrm{t}) z^{\Delta}(\mathrm{t})\right]^{\Delta}}{z^{\beta}(\delta(\mathrm{t}))}-\beta \psi(\mathrm{t}) \delta^{\Delta}(\mathrm{t}) \frac{\mathrm{r}(\sigma(\mathrm{t})) z^{\Delta}(\sigma(\mathrm{t})) z^{\Delta}(\delta(\mathrm{t}))}{z^{\beta+1}(\delta(\sigma(\mathrm{t})))} \\
& \leqslant \psi(\mathrm{t}) \frac{\left[\mathrm{r}(\mathrm{t}) z^{\Delta}(\mathrm{t})\right]^{\Delta}}{z^{\beta}(\delta(\mathrm{t}))}+\frac{\psi^{\Delta}(\mathrm{t})}{\psi(\sigma(\mathrm{t}))} \omega(\sigma(\mathrm{t}))-\beta \delta^{\Delta}(\mathrm{t}) \frac{\psi(\mathrm{t})}{\psi(\sigma(\mathrm{t}))} \frac{z^{\Delta}(\delta(\mathrm{t}))}{z(\delta(\sigma(t)))} \omega(\sigma(\mathrm{t})) .
\end{aligned}
$$

From (1.1) and (2.5) with (2.7), we obtain

$$
\omega^{\Delta}(t) \leqslant-\psi(t) q(t) Q^{\beta}(\delta(t))+\frac{\psi^{\Delta}(t)}{\psi(\sigma(t))} \omega(\sigma(t))-\beta \delta^{\Delta}(t) \frac{\psi(t)}{\psi(\sigma(t))} \frac{z^{\Delta}(\delta(t))}{z(\delta(\sigma(t)))} \omega(\sigma(t)) .
$$

Since $\left(r(t) z^{\Delta}(t)\right)$ is non-increasing, we obtain

$$
z^{\Delta}(\delta(t)) \geqslant \frac{r(\sigma(t)) z^{\Delta}(\sigma(t))}{r(\delta(t))}
$$

This with (2.8), leads to

$$
\begin{aligned}
\omega^{\Delta}(t) & \leqslant-\psi(t) q(t) Q^{\beta}(\delta(t))+\frac{\psi^{\Delta}(t)}{\psi(\sigma(t))} \omega(\sigma(t))-\beta \delta^{\Delta}(t) \frac{z^{\beta-1}(\delta(\sigma(t))) \psi(t)}{r(\delta(t)) \psi^{2}(\sigma(t))} \omega^{2}(\sigma(t)) \\
& \leqslant-\psi(t) q(t) Q^{\beta}(\delta(t))+\frac{\psi^{\Delta}(t)}{\psi(\sigma(t))} \omega(\sigma(t))-\beta M^{\beta-1} \delta^{\Delta}(t) \frac{\psi(t)}{r(\delta(t)) \psi^{2}(\sigma(t))} \omega^{2}(\sigma(t)) .
\end{aligned}
$$

Applying the inequality

$$
\mathrm{B} \omega-\mathrm{A} \omega^{\frac{\alpha+1}{\alpha}} \leqslant \frac{\alpha^{\alpha}}{(\alpha+1)^{\alpha+1}} \frac{\mathrm{B}^{\alpha+1}}{\mathrm{~A}^{\alpha}}
$$

with $B=\frac{\psi^{\Delta}(t)}{\psi(\sigma(t))}, A=\beta M^{\beta-1} \delta^{\Delta}(t) \frac{\psi(t)}{r(\delta(t)) \psi^{2}(\sigma(t))}$, we get that

$$
\omega^{\Delta}(t) \leqslant-\psi(t) q(t) Q^{\beta}(\delta(t))+\frac{1}{4} \frac{\left(\psi^{\Delta}(t)\right)^{2} r(\delta(t))}{\beta M^{(\beta-1)} \psi(t) \delta^{\Delta}(t)} .
$$

By integrating (2.9) from $t_{2}\left(t_{2} \in\left[t_{1}, \infty\right)_{\mathbb{T}}\right)$ to $t$, we have

$$
\int_{t_{2}}^{t}\left(\psi(s) q(s) Q^{\beta}(\delta(s))-\frac{1}{4} \frac{\left(\psi^{\Delta}(s)\right)^{2} r(\delta(s))}{\beta M^{(\beta-1)} \psi(s) \delta^{\Delta}(s)}\right) \Delta s \leqslant \omega\left(t_{2}\right),
$$

which contradicts (2.4). This completes the proof. 
The following theorems present oscillation criteria for (1.1) where $0<\beta<1$.

Theorem 2.6. Assume that (H1)-(H3) and (2.1) hold, and $\delta^{\Delta}>0$. For $0<\beta<1$ if the first order delay dynamic equation

$$
\omega^{\Delta}(t)+K^{\beta-1} q(t) Q^{\beta}(\delta(t)) R^{\beta}(\delta(t)) \omega(\delta(t))=0,
$$

is oscillatory for any $\mathrm{M}>0$, then (1.1) is oscillatory.

Proof. Let $x(t)$ be a nonoscillatory solution of $(1.1)$ on $\left[t_{0}, \infty\right)_{\mathbb{T}}$ such that $x(t)>0, x(\tau(t))>0$, and $x(\delta(t))>$ 0 for $t \in\left[t_{1}, \infty\right)_{\mathbb{T}}$. From (1.1), condition (2.1) and since $\left(r(t) z^{\Delta}(t)\right)$ is non-increasing, we get

$$
z(\delta(t))=z\left(t_{1}\right)+\int_{t_{1}}^{\delta(t)} \frac{r(s) z^{\Delta}(s)}{r(s)} \Delta s \geqslant r(\delta(t)) z^{\Delta}(\delta(t)) R(\delta(t)) .
$$

Proceeding as in the proof of Theorem 2.5, we get

$$
\left[r(t) z^{\Delta}(t)\right]^{\Delta} \leqslant-q(t) Q^{\beta}(\delta(t)) z^{\beta}(\delta(t)) .
$$

Since $\frac{z(t)}{R(t)}$ is decreasing, there exists a constant $K>0$ such that

$$
\frac{z(t)}{R(t)} \leqslant K, \quad t \geqslant t_{2} \geqslant t_{1} .
$$

Using (2.13) and $0<\beta<1$, then (2.12) takes the form

$$
\left(r(t) z^{\Delta}(t)\right)^{\Delta}+K^{\beta-1} q(t) Q^{\beta}(\delta(t)) R^{\beta-1}(\delta(t)) z(\delta(t)) \leqslant 0, \quad t \geqslant t_{2} \geqslant t_{1} .
$$

Using this with (2.11), we obtain

$$
\left(r(t) z^{\Delta}(t)\right)^{\Delta}+K^{\beta-1} q(t) Q^{\beta}(\delta(t)) R^{\beta}(\delta(t)) r(\delta(t)) z^{\Delta}(\delta(t)) \leqslant 0, \quad t \geqslant t_{1} .
$$

Define $\omega(t)=r(t) z^{\Delta}(t)>0$. Then from (2.14), we have

$$
\omega^{\Delta}(t)+K^{\beta-1} q(t) Q^{\beta}(\delta(t)) R^{\beta}(\delta(t)) \omega(\delta(t)) \leqslant 0, \quad t \geqslant t_{1},
$$

where $\omega(t)$, is a positive solution of the first order delay dynamic inequality (2.15). By [17, Theorem 3.1], equation (2.10) also presents a nonoscillatory solution. This contradiction proves that (1.1) is oscillatory.

Theorem 2.7. Assume that (H1)-(H3) and (2.1) hold, and $\delta^{\Delta}>0$. If $0<\beta<1$ and there exists a function $\psi(t) \in C_{r d}^{1}\left(\left[t_{0}, \infty\right)_{\mathbb{T}},(0, \infty)\right)$, such that for sufficiently large $t_{2} \geqslant t_{1}$, and for any $M>0$,

$$
\limsup _{t \rightarrow \infty} \int_{t_{2}}^{t}\left(\psi(t) q(t)\left[(1-\alpha p(\delta(t)))^{\beta}-\frac{(1-\alpha)^{\beta} p^{\beta}(\delta(t))}{M^{\beta}}\right]-\frac{1}{4} \frac{\left(\psi^{\Delta}(s)\right)^{2} r(\delta(s))}{\beta M^{(\beta-1)} \psi(s) \delta^{\Delta}(s)}\right) \Delta s=\infty,
$$

holds, then (1.1) is oscillatory.

Proof. Let $x(t)$ be a nonoscillatory solution of $(1.1)$ on $\left[t_{0}, \infty\right)_{\mathbb{T}}$ such that $x(t)>0, x(\tau(t))>0$, and $x(\delta(t))>$ 0 for $t \in\left[t_{1}, \infty\right)_{\mathbb{T}}$. From (1.1), condition (2.1), and since $z^{\Delta}(t)>0$, so there exists a constant $M>0$ such that $z(t) \geqslant M$ for all large $t \geqslant t_{1}$. From definition of $z(t)$, and by using Lemma 2.1, we get

$$
\begin{aligned}
x(t)=z(t)-p(t) x^{\alpha}(\tau(t)) & \geqslant z(t)-p(t) z^{\alpha}(\tau(t)) \\
& \geqslant z(t)-p(t) z^{\alpha}(t) \\
& \geqslant z(t)-p(t)\left(z^{\alpha}(t)-1\right)-p(t) \\
& \geqslant z(t)-\alpha p(t)(z(t)-1)-p(t) \geqslant(1-\alpha p(t)) z(t)-(1-\alpha) p(t) .
\end{aligned}
$$


This leads to

$$
(x(\delta(t))+(1-\alpha) p(\delta(t)))^{\beta} \geqslant(1-\alpha p(\delta(t)))^{\beta} z^{\beta}(\delta(t)) .
$$

Using Lemma 2.2, we get

$$
x^{\beta}(\delta(t)) \geqslant(1-\alpha p(\delta(t)))^{\beta} z^{\beta}(\delta(t))-(1-\alpha)^{\beta} p^{\beta}(\delta(t)) .
$$

From (1.1) and (2.17), we get

$$
\left(r(t) z^{\Delta}(t)\right)^{\Delta} \leqslant-q(t)(1-\alpha p(\delta(t)))^{\beta} z^{\beta}(\delta(t))+q(t)(1-\alpha)^{\beta} p^{\beta}(\delta(t)) .
$$

Define the Riccati substitution

$$
\omega(t)=\psi(t) \frac{r(t) z^{\Delta}(t)}{z^{\beta}(\delta(t))}>0 .
$$

Similarly as in the proof of Theorem 2.5 , we obtain

$$
\omega^{\Delta}(t) \leqslant \psi(t) \frac{\left[r(t) z^{\Delta}(t)\right]^{\Delta}}{z^{\beta}(\delta(\sigma(t)))}+\frac{1}{4} \frac{\left(\psi^{\Delta}(t)\right)^{2} r(\delta(t))}{\beta M^{(\beta-1)} \psi(t) \delta^{\Delta}(t)} .
$$

This with (2.18), leads to

$$
\omega^{\Delta}(t) \leqslant-\psi(t) q(t)\left((1-\alpha p(\delta(t)))^{\beta}-\frac{(1-\alpha)^{\beta} p^{\beta}(\delta(t))}{M^{\beta}}\right)+\frac{1}{4} \frac{\left(\psi^{\Delta}(t)\right)^{2} r(\delta(t))}{\beta M^{(\beta-1)} \psi(t) \delta^{\Delta}(t)} .
$$

By integrating (2.19) from $t_{2}\left(t_{2} \in\left[t_{1}, \infty\right) \mathbb{T}\right)$ to $t$, we have

$$
\int_{t_{2}}^{t}\left(\psi(t) q(t)\left[(1-\alpha p(\delta(t)))^{\beta}-\frac{(1-\alpha)^{\beta} p^{\beta}(\delta(t))}{M^{\beta}}\right]-\frac{1}{4} \frac{\left(\psi^{\Delta}(s)\right)^{2} r(\delta(s))}{\beta M^{(\beta-1)} \psi(s) \delta^{\Delta}(s)}\right) \Delta s \leqslant \omega\left(t_{2}\right),
$$

which contradicts (2.16). This completes the proof.

Now, we consider

$$
\int_{\mathrm{t}_{0}}^{\infty} \frac{1}{\mathrm{r}(\mathrm{s})} \Delta \mathrm{s}<\infty
$$

First, we establish an oscillation criterion when $\beta \geqslant 1$.

Theorem 2.8. Let $\beta \geqslant 1$. Assume that (H1)-(H3) and (2.20) hold, and $\lim _{\mathrm{t} \rightarrow \infty} \mathrm{p}(\mathrm{t})=0$. If

$$
\limsup _{t \rightarrow \infty} \int_{t_{2}}^{t}\left(\varphi(s) \hat{R}(\sigma(s))-\frac{1}{4 \beta C^{\beta-1} r(s) \hat{R}^{\beta}(\sigma(s))}\right) \Delta s=\infty,
$$

holds for any $\mathrm{C}>0$ and sufficiently large $\mathrm{t}_{2} \geqslant \mathrm{t}_{1}$, then (1.1) is oscillatory.

Proof. Let $x(t)$ be a nonoscillatory solution of $(1.1)$ on $\left[t_{0}, \infty\right)_{\mathbb{T}}$ such that $x(t)>0, x(\tau(t))>0$, and $x(\delta(t))>$ 0 for $\mathrm{t} \in\left[\mathrm{t}_{1}, \infty\right)_{\mathbb{T}}$. From (1.1) and condition (2.20), we get

$$
\left(r(t) z^{\Delta}(t)\right)^{\Delta} \leqslant 0,
$$

which means that $\left(\mathrm{r}(\mathrm{t}) z^{\Delta}(\mathrm{t})\right)$ is non-increasing and implies two cases: case (I) $z^{\Delta}(\mathrm{t})>0$, or case (II) $z^{\Delta}(t)<0$. Therefor $\lim _{t \rightarrow \infty} z(t)=\lim _{t \rightarrow \infty} x(t)+p(t) x^{\alpha}(\tau(t))=\lim _{t \rightarrow \infty} x(t)$, since $\lim _{t \rightarrow \infty} p(t)=0$.

Now, we consider case (I). Since $z^{\Delta}(t)>0$, then there exists a constant $d>0$, such that

$$
x(t) \geqslant x(\tau(t))>d, \quad \text { for } t \geqslant t_{1},
$$

from (1.1), (2.23), we get

$$
\left(r(t) z^{\Delta}(t)\right)^{\Delta}+q(t) d^{\beta}<0, \quad t \geqslant t_{1} .
$$


Integrating (2.24) from $t_{1}$ to $t$, we get

$$
r(t) z^{\Delta}(t)-r\left(t_{1}\right) z^{\Delta}\left(t_{1}\right)+d^{\beta} \int_{t_{1}}^{t} q(s) \Delta s<0 .
$$

But $\int_{\mathrm{t}_{1}}^{\infty} \mathrm{q}(\mathrm{s}) \Delta \mathrm{s}=\infty$ which with (2.25) leads to

$$
\lim _{t \rightarrow \infty} r(t) z^{\Delta}(t)=-\infty,
$$

which is a contradiction with the eventual positivity of $\left(r(t) z^{\Delta}(t)\right)$.

Next, we consider case (II). Define

$$
\omega(t)=\frac{r(t) z^{\Delta}(t)}{z^{\beta}(t)} .
$$

Then $\omega(t)<0$ for $t \geqslant t_{1}$. From (2.22), we get

$$
z^{\Delta}(u) \leqslant \frac{r(t)}{r(u)} z^{\Delta}(t), \quad u>t \in\left[t_{1}, \infty\right)_{\mathbb{T}}
$$

By integrating from $t$ to $j$, we get

$$
z(\mathfrak{j})-z(\mathrm{t}) \leqslant \mathrm{r}(\mathrm{t}) z^{\Delta}(\mathrm{t}) \int_{\mathrm{t}}^{\mathrm{j}} \frac{1}{\mathrm{r}(\mathrm{u})} \Delta \mathrm{u}
$$

and by letting $j \rightarrow \infty$, leads to

$$
-1 \leqslant \frac{r(t) z^{\Delta}(t)}{z(t)} \hat{R}(t)
$$

with definition of $\omega(t)$, we get

$$
\omega(t) \hat{R}(t) \geqslant-L^{1-\beta}, \quad \text { where } L=z\left(t_{1}\right)>0 .
$$

On the other hand, we can use (2.26) to obtain

$$
\left(\frac{z(t)}{\hat{R}(t)}\right)^{\Delta}=\frac{z^{\Delta}(t) \hat{R}(t)-z(t) \hat{R}^{\Delta}(t)}{\hat{R}(t) \hat{R}(\sigma(t))} \geqslant \frac{z^{\Delta}(t) \hat{R}(t)+\frac{z(t)}{r(t)}}{\hat{R}(t) \hat{R}(\sigma(t))} \geqslant 0,
$$

so,

$$
\frac{z(\sigma(t))}{\hat{R}(\sigma(t))} \geqslant \frac{z(\tau(t))}{\hat{R}(\tau(t))}
$$

Since $z(t):=x(t)+p(t) x^{\alpha}(\tau(t))$, we get

$$
x(t)=z(t)-p(t) x^{\alpha}(\tau(t)) \geqslant z(t)-p(t) z^{\alpha}(\tau(t)) .
$$

This with (2.28) and taking into account $z^{\Delta}(\mathrm{t})<0$, leads to

$$
x(t) \geqslant z(t)-p(t) \frac{\hat{R}^{\alpha}(\tau(t))}{\hat{R}^{\alpha}(\sigma(t))} z^{\alpha}(\sigma(t)) \geqslant\left(1-p(t) \frac{\hat{R}^{\alpha}(\tau(t))}{\hat{R}^{\alpha}(\sigma(t))} z^{\alpha-1}(\sigma(t))\right) z(t) .
$$

Since $\left(\frac{z(t)}{\hat{R}(t)}\right)$ is positive and increasing, we get

$$
\frac{z(\sigma(t))}{\hat{R}(\sigma(t))} \geqslant \frac{z(t)}{\hat{R}(t)} \geqslant \frac{z\left(t_{1}\right)}{\hat{R}\left(t_{1}\right)}=C>0, \quad t \geqslant t_{1},
$$




$$
z(\sigma(t)) \geqslant C \hat{R}(\sigma(t)), \quad t \geqslant t_{1}
$$

From (2.29) and (2.31), we get

$$
x(t) \geqslant\left(1-C^{\alpha-1} p(t) \frac{\hat{R}^{\alpha}(\tau(t))}{\hat{R}(\sigma(t))}\right) z(t)=P(t) z(t) .
$$

From (1.1) and (2.32), we get

$$
\begin{aligned}
\left(r(t) z^{\Delta}(t)\right)^{\Delta} & =-q(t) x^{\beta}(\delta(t)) \\
& \leqslant-q(t) P^{\beta}(\delta(t)) z^{\beta}(\delta(t)) \\
& \leqslant-q(t) P^{\beta}(\delta(t)) z^{\beta}(t) \\
& \leqslant-q(t) P^{\beta}(\delta(t)) z^{\beta}(\sigma(t)):=-\varphi(t) z^{\beta}(\sigma(t)) .
\end{aligned}
$$

Now, from definition of $\omega(t)$, we get

$$
\omega^{\Delta}(\mathrm{t})=\frac{\left(\mathrm{r}(\mathrm{t}) z^{\Delta}(\mathrm{t})\right)^{\Delta} z^{\beta}(\mathrm{t})-r(\mathrm{t}) z^{\Delta}(\mathrm{t})\left(z^{\beta}(\mathrm{t})\right)^{\Delta}}{z^{\beta}(\mathrm{t}) z^{\beta}(\sigma(t))} \leqslant \frac{\left(r(t) z^{\Delta}(\mathrm{t})\right)^{\Delta}}{z^{\beta}(\sigma(t))}-\beta \frac{r(\mathrm{t})\left(z^{\Delta}(\mathrm{t})\right)^{2}}{z(\mathrm{t}) z^{\beta}(\sigma(t))} .
$$

Also, since $z^{\Delta}(t)<0$ this with (2.30) and (2.34), we obtain

$$
\omega^{\Delta}(t) \leqslant \frac{\left(r(t) z^{\Delta}(t)\right)^{\Delta}}{z^{\beta}(\sigma(t))}-\beta \frac{r(t)\left(z^{\Delta}(t)\right)^{2}}{z^{\beta+1}(t)} \leqslant \frac{\left(r(t) z^{\Delta}(t)\right)^{\Delta}}{z^{\beta}(\sigma(t))}-\beta C^{\beta-1} \frac{\hat{R}^{\beta-1}(t)}{r(t)} \omega^{2}(t) .
$$

By using the last inequality with (2.33), we conclude that

$$
\omega^{\Delta}(t) \leqslant-\varphi(t)-\beta C^{\beta-1} \frac{\hat{R}^{\beta-1}(\sigma(t))}{r(t)} \omega^{2}(t) .
$$

By using (2.35), we obtain the following

$$
(\hat{R}(t) \omega(t))^{\Delta}=\hat{R}^{\Delta}(t) \omega(t)+\hat{R}(\sigma(t)) \omega^{\Delta}(t) \leqslant \hat{R}^{\Delta}(t) \omega(t)-\varphi(t) \hat{R}(\sigma(t))-\beta C^{\beta-1} \frac{\hat{R}^{\beta}(\sigma(t))}{r(t)} \omega^{2}(t) .
$$

Applying the inequality

$$
\mathrm{B} \omega-\mathrm{A} \omega^{\frac{\alpha+1}{\alpha}} \leqslant \frac{\alpha^{\alpha}}{(\alpha+1)^{\alpha+1}} \frac{\mathrm{B}^{\alpha+1}}{\mathrm{~A}^{\alpha}}
$$

with $B=\hat{R}^{\Delta}(t), \quad A=\beta C^{\beta-1} \frac{\hat{R}^{\beta}(\sigma(t))}{r(t)}$, where $\alpha=1$, we get that

$$
(\hat{R}(t) \omega(t))^{\Delta} \leqslant-\varphi(t) \hat{R}(\sigma(t))+\frac{r(t)\left(\hat{R}^{\Delta}(t)\right)^{2}}{4 \beta C^{\beta-1} \hat{R}^{\beta}(\sigma(t))} \leqslant-\varphi(t) \hat{R}(\sigma(t))+\frac{1}{4 \beta C^{\beta-1} r(t) \hat{R}^{\beta}(\sigma(t))} .
$$

By integrating (2.36) from $t_{2} \in\left[t_{1}, \infty\right)_{\mathbb{T}}$ to $t$, in view of $(2.27)$ we have

$$
\int_{t_{2}}^{t}\left(\varphi(s) \hat{R}(\sigma(s))-\frac{1}{4 \beta C^{\beta-1} r(s) \hat{R}^{\beta}(\sigma(s))}\right) \Delta s \leqslant L^{1-\beta}+\hat{R}\left(t_{2}\right) \omega\left(t_{2}\right)<\infty,
$$

which contradicts (2.21). This completes the proof.

Letting $\beta=1$, Theorem 2.8 yields the following result. 
Corollary 2.9. Let $\beta=1$. Assume that (H1)-(H3) and (2.20) hold, and $\lim _{\mathrm{t} \rightarrow \infty} \mathrm{p}(\mathrm{t})=0$. If

$$
\limsup _{t \rightarrow \infty} \int_{t_{2}}^{t}\left(q(s) P(\delta(s)) \hat{R}(\sigma(s))-\frac{1}{4 r(s) \hat{R}(\sigma(s))}\right) \Delta s=\infty,
$$

holds for sufficiently large $\mathrm{t}_{2} \geqslant \mathrm{t}_{0}$, then (1.1) is oscillatory.

Now, we establish an oscillation criterion when $0<\beta<1$.

Theorem 2.10. Let $0<\alpha<1$ and $0<\beta<1$. Assume that (H1)-(H3) and (2.20) hold, and $\lim _{\mathrm{t} \rightarrow \infty} \mathrm{p}(\mathrm{t})=0$. If

$$
\limsup _{t \rightarrow \infty} \int_{t_{2}}^{t}\left(\operatorname{Lq}(s) P^{\beta}(s) \hat{R}(\sigma(s))-\frac{1}{4 r(s) \hat{R}(\sigma(s))}\right) \Delta s=\infty,
$$

holds for some $\mathrm{L}:=\mathrm{K}^{\beta-1}>0$ and sufficiently large $\mathrm{t}_{2} \geqslant \mathrm{t}_{0}$, then (1.1) is oscillatory.

Proof. Let $x(t)$ be a nonoscillatory solution of $(1.1)$ on $\left[t_{0}, \infty\right)_{\mathbb{T}}$ such that $x(t)>0, x(\tau(t))>0$, and $x(\delta(t))>$ 0 for $t \in\left[t_{1}, \infty\right)_{\mathbb{T}}$. From (1.1) and condition (2.20), we have two cases: case (I) $z^{\Delta}(t)>0$, or case (II) $z^{\Delta}(\mathrm{t})<0$.

Case (I) is similar as in the proof of Theorem 2.8.

Now, we consider case (II). Since $z(t)<0$, and $\lim _{t \rightarrow \infty} z(t)=\lim _{t \rightarrow \infty} x(t)$, then we have two possibilities: either $\lim _{t \rightarrow \infty} z(t)=d_{1}>0$ or $\lim _{t \rightarrow \infty} z(t)=0$. First case implies that $\lim _{t \rightarrow \infty} x(t)=d_{1}$, so there exists a constant $d_{2}>0$ such that $x(t)>d_{2}$, which leads to a contradiction as in case (I). Now, for the second case we define

$$
\omega(t)=\frac{r(t) z^{\Delta}(t)}{z(t)}<0 .
$$

This case implies that

$$
0<z(t)<N \text {, where } N:=L^{1 /(\beta-1)}, \text { for } t \geqslant t_{1} \text {. }
$$

Now, proceeding as in the proof of Theorem 2.8, from (2.33) and (2.39), we obtain that

$$
\begin{aligned}
\left(r(t) z^{\Delta}(t)\right)^{\Delta} \leqslant-q(t) P^{\beta}(t) z^{\beta}(t) & \leqslant-q(t) P^{\beta}(t) z^{\beta-1}(t) z(\sigma(t)) \\
& \leqslant-q(t) P^{\beta}(t) N^{\beta-1} z(\sigma(t))=-L q(t) P^{\beta}(t) z(\sigma(t)) .
\end{aligned}
$$

Now, as similar as in Theorem 2.8, we get

$$
(\hat{R}(t) \omega(t))^{\Delta} \leqslant-L q(t) P^{\beta}(t) \hat{R}(\sigma(t))+\frac{1}{4 r(t) \hat{R}(\sigma(t))} .
$$

By integrating (2.40) from $t_{2}\left(t_{2} \in\left[t_{1}, \infty\right)_{\mathbb{T}}\right)$ to $t$, in view of $(2.26)$ we have

$$
\int_{t_{2}}^{t}\left(L q(s) P^{\beta}(s) \hat{R}(\sigma(s))-\frac{1}{4 r(s) \hat{R}(\sigma(s))}\right) \Delta s \leqslant 1+\hat{R}\left(t_{2}\right) \omega\left(t_{2}\right)<\infty,
$$

which contradicts (2.38). This completes the proof.

\section{Examples}

Example 3.1 ([25]). Assume $\mathbb{T}=\mathbb{R}$. Consider the second order neutral differential equation

$$
\left(t\left(x(t)+\frac{1}{t} x^{1 / 3}(t / 2)\right)^{\prime}\right)^{\prime}+t x^{3}(t / 2)=0, \quad t \geqslant 1
$$


where $\alpha=1 / 3, \beta=3, r(t)=t, p(t)=\frac{1}{t}, q(t)=t, \tau(t)=\frac{t}{2}$, and $\delta(t)=\frac{t}{2}$. It is clear that $\int_{t_{0}}^{\infty} \frac{1}{r(s)} d s=$ $\int_{1}^{\infty} \frac{1}{s} \mathrm{ds}=\infty$, then for $M=1$ and $\psi(t)=t$ we can apply condition (2.4) that becomes

$$
\limsup _{t \rightarrow \infty}\left[\int_{1}^{t}\left(s^{2}\left(1-\frac{2}{s}\right)^{3}-\frac{\log (s / 2)}{6 s}\right) d s\right]=\infty .
$$

So it is clear that (3.1) satisfies all conditions of Theorem 2.5, then equation (3.1) is oscillatory.

Example 3.2. Assume $\mathbb{T}=\mathbb{R}$. Consider the second order neutral differential equation

$$
\left(x(t)+\frac{1}{t} x^{1 / 3}(t / 2)\right)^{\prime \prime}+\frac{a}{t^{4 / 3}} x^{1 / 3}(t / 2)=0, \quad t \geqslant 0,
$$

where $\alpha=\beta=1 / 3, r(t)=1, p(t)=\frac{1}{t}, q(t)=\frac{a}{t^{4 / 3}}$, and $\tau(t)=\delta(t)=\frac{t}{2}$. It is clearly that $\int_{t_{0}}^{\infty} \frac{1}{r(s)} d s=$ $\int_{0}^{\infty} \mathrm{ds}=\infty$, then for $M=1$ and $\psi(t)=t$ we can apply condition (2.16) that becomes

$$
\limsup _{t \rightarrow \infty} \int_{t_{2}}^{t}\left(\frac{a}{t^{1 / 3}}\left[\left(1-\frac{2}{3 t}\right)^{1 / 3}-\left(\frac{4}{3 t}\right)^{1 / 3}\right]-\frac{3}{2 t}\right) d s=\infty,
$$

for $a>0$, it is clear that equation (3.2) satisfies all conditions of Theorem 2.7, then equation (3.2) is oscillatory for $a>0$.

Example 3.3 ([12]). Assume $\mathbb{T}=\mathbb{Z}$. Consider the second order neutral difference equation

$$
\Delta\left((n+1) \Delta\left(x_{n}+\frac{1}{n} x_{n-2}^{1 / 3}\right)\right)+\left(4 n+10+\frac{2 n+1}{n(n+1)}\right) x_{n-3}^{3}=0, \quad n \geqslant 1,
$$

where $\alpha=1 / 3, \beta=3, r_{n}=n+1, p_{n}=\frac{1}{n}, q_{n}=4 n+10+\frac{2 n+1}{n(n+1)}, \tau_{n}=n-2$, and $\delta_{n}=n-3$. It is clearly that $\sum_{\mathrm{t}_{0}}^{\infty} \frac{1}{\mathrm{r}(\mathrm{s})}=\sum_{1}^{\infty} \frac{1}{\mathrm{~s}+1}=\infty$, then for $M=1$ and $\psi_{\mathrm{n}}=1$ we can apply condition (2.4) that becomes

$$
\limsup _{n \rightarrow \infty}\left[\sum_{s=1}^{n}\left(\left(4 s+10+\frac{2 s+1}{s(s+1)}\right)\left(1-\frac{1}{s}\left(\frac{R_{s+1}}{R_{s}}\right)^{1 / 3}\right)^{3}\right)\right]=\infty .
$$

So it is clear that (3.3) satisfies all conditions of Theorem 2.5, then equation (3.3) is oscillatory.

Example 3.4 ([25]). Assume $\mathbb{T}=\mathbb{R}$. Consider the second order neutral differential equation

$$
\left(t^{2}\left(x(t)+\frac{p_{0}}{t^{2-2 \alpha}} x^{\alpha}\left(\frac{t}{2}\right)\right)^{\prime}\right)^{\prime}+\lambda x(t)=0, \quad t \geqslant 1
$$

where $p_{0}>0, \lambda>0$, and $0<\alpha<1$. Here, we have $\beta=1, r(t)=t^{2}, p(t)=\frac{p_{0}}{t^{2-2 \alpha}}, q(t)=\lambda, \tau(t)=\frac{t}{2}$, and $\delta(t)=t$. It is clearly that $\int_{t_{0}}^{\infty} \frac{1}{r(s)} d s=\int_{1}^{\infty} \frac{1}{s^{2}} d s<\infty$, then for $C=1$ we can apply condition (2.37) that becomes

$$
\limsup _{t \rightarrow \infty}\left[\int_{1}^{t}\left(\frac{\lambda}{s}\left(1-\frac{p_{0}}{t^{2-2 \alpha}} \frac{\left(\frac{2}{s}\right)^{\alpha}}{\left(\frac{1}{s}\right)}\right)-\frac{1}{4 s}\right) d s\right]=(4 \lambda-1) \infty .
$$

So it is clear that (3.4) satisfies all conditions of Corollary 2.9 for $\lambda>\frac{1}{4}$, then for $\lambda>\frac{1}{4}$ equation (3.4) is oscillatory. 
Example 3.5 ([4]). Assume $\mathbb{T}=\mathbb{Z}$. Consider the second order neutral difference equation

$$
\Delta\left(n(n+1) \Delta^{m-1}\left(x_{n}+\frac{1}{n} x_{n-2}^{1 / 3}\right)\right)+n x_{n-1}^{1 / 3}=0, \quad n \geqslant 2,
$$

where $m=2, \alpha=\beta=1 / 3, r_{n}=n(n+1), p_{n}=\frac{1}{n}, q_{n}=n, \tau_{n}=n-2$, and $\delta_{n}=n-1$. It is clear that $\sum_{\mathrm{t}_{0}}^{\infty} \frac{1}{\mathrm{r}(\mathrm{s})}=\sum_{2}^{\infty} \frac{1}{\mathrm{~s}(\mathrm{~s}+1)}<\infty$, then for $\mathrm{L}=1$ we can apply condition (2.38) that becomes

$$
\limsup _{n \rightarrow \infty}\left[\sum_{s=2}^{n}\left(s\left(1-\frac{1}{s} \frac{\left(\frac{1}{s-2}\right)^{1 / 3}}{\left(\frac{1}{s+1}\right)}\right)^{1 / 3}-\frac{1}{4 s}\right)\right]=\infty \text {. }
$$

So it is clear that (3.5) satisfies all conditions of Theorem 2.10, then equation (3.5) is oscillatory.

Remark 3.1. It would be interesting to establish sufficient conditions for the oscillatory behavior of solutions to (1.1) when $z(t)=x(t)-p(t) x^{\alpha}(\tau(t))$. Many studies have been devoted to the oscillatory behavior of solutions to different classes of equations with negative neutral coefficients, see [9, 11, 22].

\section{Acknowledgment}

The authors express their sincere gratitude to the anonymous referee for careful reading of the original manuscript and useful comments that helped to improve presentation of results and accentuate important details.

\section{References}

[1] R. P. Agarwal, M. Bohner, T. Li, C. Zhang, Oscillation of second-order differential equations with a sublinear neutral term, Carpathian J. Math., 30 (2014), 1-6. 1, 1

[2] R. P. Agarwal, M. Bohner, T. Li, C. Zhang, Oscillation of second-order Emden-Fowler neutral delay differential equations, Ann. Mat. Pura Appl., 193 (2014), 1861-1875. 1

[3] R. P. Agarwal, D. O’Regan, S. H. Saker, Oscillation criteria for second-order nonlinear neutral delay dynamic equations, J. Math. Anal. Appl., 300 (2004), 203-217. 1

[4] G. Ayyappan, G. Nithyakala, Some oscillation results for even order delay difference equations with a sublinear neutral term, Abstr. Appl. Anal., 2018 (2018), 7 pages. 1, 3.5

[5] B. Baculíková, J. Džurina, Oscillation of third-order neutral differential equations, Math. Comput. Modelling, 52 (2010), 215-226. 1

[6] M. Bohner, H. A. El-Morshedy, S. R. Grace, I. Sağer, Oscillation of second-order nonlinear difference equations with sublinear neutral term, Math. Morav., 23 (2019), 1-10. 1

[7] M. Bohner, S. Grace, I. Jadlovská, Oscillation criteria for second-order neutral delay differential equations, Electron. J. Qual. Theory Differ. Equ., 2017 (2017), 1-12. 1

[8] M. Bohner, T. S. Hassan, T. Li, Fite-Hille-Wintner-type oscillation criteria for second-order half-linear dynamic equations with deviating arguments, Indag. Math., 29 (2018), 548-560. 1

[9] M. Bohner, T. Li, Oscillation of second-order p-Laplace dynamic equations with a nonpositive neutral coefficient, Appl. Math. Lett., 37 (2014), 72-76. 3.1

[10] M. Bohner, A. Peterson, Dynamic equations on time scales: An introduction with applications, Birkhäuser, Boston, (2001). 2.3

[11] G. E. Chatzarakis, S. R. Grace, I. Jadlovská, T. Li, E. Tunç, Oscillation criteria for third-order emden-fowler differential equations with unbounded neutral coefficients, Complexity, 2019 (2019), 8 pages. 3.1

[12] C. Dharuman, R. J. Graef, E. Thandapani, S. K. Vidhyaa, Oscillation of second order difference equation with a sub-linear neutral term, J. Math. Appl., 40 (2017), 59-67. 1, 1, 3.3

[13] J. Džurina, S. R. Grace, I. Jadlovská, T. Li, Oscillation criteria for second-order Emden-Fowler delay differential equations with a sublinear neutral term, Math. Nachr., 293 (2020), 910-922. 1

[14] J. Dzurina, E. Thandapani, B. Baculikova, C. Dharuman, N. Prabaharan, Oscillation of Second Order Nonlinear Differential Equations with Several Sub-Linear Neutral Terms, Nonlinear Dyn. Syst. Theory, 19 (2019), $124-132$.

[15] L. Erbe, T. S. Hassan, A. Peterson, Oscillation criteria for nonlinear functional neutral dynamic equations on time scales, J. Difference Equ. Appl., 15 (2009), 1097-1116. 
[16] S. R. Grace, I. Jadlovská, A. Zafer, On Oscillation of Second Order Delay Differential Equations with a Sublinear Neutral Term, Mediterr. J. Math., 17 (2020), 1-11. 1, 1

[17] B. Karpuz, Ö. Öcalan, New oscillation tests and some refinements for first-order delay dynamic equations, Turkish J. Math., 40 (2016), 850-863. 2

[18] T. Li, R. P. Agarwal, M. Bohner, Some oscillation results for second-order neutral dynamic equations, Hacet. J. Math. Stat, 41 (2012), 715-721. 1

[19] T. Li, N. Pintus, G. Viglialoro, Properties of solutions to porous medium problems with different sources and boundary conditions, Z. Angew. Math. Phys., 70 (2019), 1-18. 1

[20] T. Li, Y. V. Rogovchenko, Oscillation of second-order neutral differential equations, Math. Nachr., 288 (2015), 1150-1162.

[21] T. Li, Y. V. Rogovchenko, Oscillation criteria for second-order superlinear Emden-Fowler neutral differential equations, Monatsh. Math., 184 (2017), 489-500. 1

[22] T. Li, Y. V. Rogovchenko, On the asymptotic behavior of solutions to a class of third-order nonlinear neutral differential equations, Appl. Math. Lett., 105 (2020), 7 pages. 3.1

[23] T. Li, S. H. Saker, A note on oscillation criteria for second-order neutral dynamic equations on isolated time scales, Commun. Nonlinear Sci. Numer. Simul., 19 (2014), 4185-4188. 1

[24] T. Li, C. Zhang, E. Thandapani, Asymptotic behavior of fourth-order neutral dynamic equations with noncanonical operators, Taiwanese J. Math., 18 (2014), 1003-1019. 1

[25] S. Tamilvanan, E. Thandapani, J. Dzurina, Oscillation of second order nonlinear differential equation with sublinear neutral term, Differ. Equ. Appl., 9 (2017), 29-35. 1, 3.1, 3.4

[26] E. Thandapani, M. Vijaya, T. Li, On the oscillation of third order half-linear neutral type difference equations, Electron. J. Qual. Theory Differ. Equ., 2011 (2011), 1-13. 2.1, 2.2

[27] C. Zhang, R. P. Agarwal, M. Bohner, T. Li, Oscillation of second-order nonlinear neutral dynamic equations with noncanonical operators, Bull. Malays. Math. Sci. Soc., 38 (2015), 761-778. 1 